This item was submitted to Loughborough's Research Repository by the author.

Items in Figshare are protected by copyright, with all rights reserved, unless otherwise indicated.

\title{
Ethnographic action research: media, information and communicative ecologies for development initiatives
}

\section{PLEASE CITE THE PUBLISHED VERSION}

https://uk.sagepub.com/en-gb/eur/the-sage-handbook-of-action-research/book242797

\section{PUBLISHER}

(c) SAGE Publications

\section{VERSION}

AM (Accepted Manuscript)

\section{PUBLISHER STATEMENT}

This work is made available according to the conditions of the Creative Commons Attribution-NonCommercialNoDerivatives 4.0 International (CC BY-NC-ND 4.0) licence. Full details of this licence are available at: https://creativecommons.org/licenses/by-nc-nd/4.0/

\section{LICENCE}

CC BY-NC-ND 4.0

\section{REPOSITORY RECORD}

Tacchi, Jo. 2019. "Ethnographic Action Research: Media, Information and Communicative Ecologies for Development Initiatives”. figshare. https://hdl.handle.net/2134/23781. 


\section{Ethnographic action research: Media, information and}

\section{communicative ecologies for development initiatives}

\section{Jo Tacchi}

This chapter introduces ethnographic action research (EAR). EAR is a project development methodology that was designed specifically for media and information and communication technology for development (ICT4D) initiatives. It provides a set of tools for developing an ICT4D initiative taking into account its local communicative and social contexts and relationships. First developed in 2002, continuous testing and refinement of EAR has taken place in projects conducted in various South and South East Asian countries. Most recently it has been further developed into a participatory monitoring and evaluation methodology (Lennie and Tacchi, 2013).

Ethnographic action research is a form of participatory action research (PAR). The ethnographic approach combined with action research means that it builds upon notions of immersion, long term engagement, and understanding local contexts holistically. It was initially designed to help project's develop and adapt to local situations, and in this sense it is a form of developmental evaluation (Patton, 2011). It draws on key ethnographic methods such as participant observation and in-depth interviews, and takes a multi-method approach. A foundational concept of EAR is communicative ecologies, which involves paying attention to wider contexts of information and communication flows and channels, formal and informal, technical and social, to understand communication opportunities and barriers. 
It is important to understand where EAR came from, and the central importance of the communicative ecologies concept. EAR and communicative ecologies responded to (at least) two central organizing concepts or categories in the ICT4D field. First, there is the technological determinist and modernizing ideas that underpin development whereby technology is a key to development itself (see Tacchi, forthcoming and Slater, 2013). Second there is the dominance of measurement and impact, what is now widely known as 'results based management' (see Lennie and Tacchi, 2013). EAR and communicative ecologies challenged both by taking the position that, if we start by considering how people communicate around certain themes, or to accomplish certain tasks, we can start to appreciate which channels and flows are used and why, how this relates to other possible uses, and therefore where there are opportunities and barriers to an ICT4D initiative. In other words, there was, and remains, a need to challenge development assumptions about both development and media and communication technologies and to embed our understandings of both within broader social structures and relationships (see Slater, 2013).

In this chapter we describe the key characteristics of EAR and communicative ecologies, and how they have been combined and put into practice through a few specific ICT4D projects, with full recognition and indeed the intention that they might be taken up and adapted by others in various other contexts and combinations for a range of different purposes (as they have been). We first explore the background to the development of EAR through two communication research projects, the first in Sri Lanka (the KCRIP study) and the second in sites across South Asia (the ictPR project). We then outline its core components and explain communicative ecologies. We explore EAR's application and further development through a project in South and South East Asia (the Finding a Voice research project). Next we look at the adaptation of EAR from a project development methodology to a participatory monitoring and evaluation methodology in Nepal (the Assessing 
Communication for Social Change research project). There are two key online training resources available and these will be discussed in the conclusion.

\section{Background and development}

\section{Where EAR came from: the KCRIP study}

EAR was first developed in 2002 in response to a need to find new ways of understanding the implications of ICT for development. At the time there was major investment in a range of ICT4D activities through, for example, large telecentre or multimedia centre programmes (see http://www.telecentre.org and http://www.unesco.org/webworld/cmc). What was missing was a way of understanding the impact of such initiatives, because standard and mainstream monitoring and evaluation and impact assessments were missing what was anecdotally reported as impactful through local success stories. To use Cedric Price's well repeated phrase: technology is the answer, but what was the question? Mainstream approaches tended to focus on predetermined indicators of change brought about by the introduction of new ICTs, and yet anecdotes talked of rather more unexpected outcomes, the kinds of things that it is hard to predict or perhaps only tangentially relate to the technology itself. For example, Slater (2013) writing of the design of the survey for the KCRIP study, realized after entering the field and conducting the ethnography that the very concepts and categories that structured the survey needed to be rethought and reframed - we were asking the wrong questions. We considered that ethnography with its concern to take nothing for granted and to privileged local perspectives and experiences, might help us understand in a more nuanced way the kinds of changes that surveys and impact assessments were failing to capture.

The British Government's Department for International Development (DFID) funded a research project in Sri Lanka to explore the potential of ethnography to better understand ICT 
interventions. Also supported by UNESCO, the intention was to develop a transferable methodology for understanding and evaluating ICT4D initiatives (Slater, Tacchi and Lewis, 2002). The ethnographic study was of the Kothmale Community Radio and Internet Project (KCRIP) in Central Province, Sri Lanka. The Kothmale community radio station had been operating since the 1980s, and the Internet centre since 2000. KCRIP was UNESCO's first 'community multimedia centre' and it was anecdotally having a lot of positive outcomes, but little rigorous research and no regular monitoring and evaluation was taking place to back this up. The combination of the Internet centre and the radio station was of particular interest in an area where, at that time, most people had access to radio, but very few to other communication technologies such as telephones, computers and the Internet.

We used some core ethnographic methods including participant observation and in-depth interviews in a range of locations (including households, shops, temples, the radio station and computer centre, and local schools). We also undertook a survey administered in 200 households. Taken together, this research reported some interesting findings about the activities of KCRIP community radio project and some of the characteristics of local communities and their media uses and information sources (Slater, Tacchi and Lewis 2002).

What it failed to do was give us a methodology that was useful for the ongoing development of KCRIP community radio itself, and a transferable methodology for the development and evaluation of ICT4D projects more broadly. While the 'ethnographic evaluation' of KCRIP came up with interesting and important findings in terms of how the project might adapt and develop, there was a significant limitation that directly led to the development of ethnographic action research. Rather than simply coming up with research findings and recommendations, we wanted those involved in KCRIP community radio to be able to apply them in ways that helped them to achieve their objectives. Despite enormous 
interest from KCRIP staff and volunteers in our research, there was no real ownership of the findings on the part of KCRIP, and no obvious route to making use of them. As we were about to leave, we realized that a different application of an ethnographic approach would be necessary here, if it was to be useful and useable by KCRIP and other ICT4D initiatives. We recognized the need to develop a methodology that integrated an ethnographic approach with participatory action research. We wanted to develop a methodology specifically for ICT4D which project workers themselves could use to help their initiatives develop effectively in their local settings, encompassing rich understandings of local conditions and needs. These understandings could inform project development through ongoing planning, action and reflection.

\section{Testing EAR: the ictPR project}

While the KCRIP study led to the development of EAR, it was not yet fully developed and tested. It provided the end point of the KCRIP radio study, and the starting point for the research component of UNESCO's ICT for Poverty Reduction (ictPR) project undertaken throughout 2003. The ictPR project aimed to innovate and research the use of information and communication technology ICTs for poverty reduction in nine community-based media and ICT centres in India, Nepal, Bangladesh, Sri Lanka and Bhutan (Slater and Tacchi, 2004). Each centre had a different configuration of media and technology with different objectives - some focused on building information sharing networks for rural women, others on ICT centres for youth; some developed computer centres while others linked computers and the Internet to TV and/or radio. Research was built into the development of ictPR poverty reduction efforts from the start, and each centre appointed its own local researcher. Here is the first major shift from the KCRIP radio study to the implementation of EAR in ictPR poverty reduction. We trained local researchers who were embedded in the local initiatives to be ethnographic action researchers. 
We held training workshops and visited many of the research sites to work alongside the researchers and train them in the process. These local researchers collected and shared information on their initiatives, which helped us understand some of the uses and possibilities of traditional and new technologies for development, but more importantly, helped each local initiative improve their own practices. They used the research to inform their ongoing development, following and repeating an action research cycle of plan, do, reflect. Through this process we developed a training handbook (Tacchi, Slater and Hearn, 2003) that was later further refined and developed through the Finding a Voice project - described later in this chapter - into an online training resource, http://ear.findingavoice.org.

\section{Core concepts and components}

\section{Communicative ecologies}

The application of EAR to a media, communication or ICT4D initiative requires an understanding of the concept of communicative ecologies. Communicative ecologies refer to the complex systems of communication, media and information flows in a community, or as Slater (2013) puts it, and drawing on actor network theory (ANT), a communicative ecology is made up of communicative assemblages. It does not assume a hierarchy of ways of communicating, but provides a way of thinking from scratch about the ways in which information and communication flow between people and through infrastructures, including not just electronic media channels but also roads and busses, and any other communication processes and practices that are significant in a particular context. From the KCRIP study we learned that radio and Internet were just two of the many ways of communicating and sharing information in this area. The importance of information sharing (or gossip) at wells, and the very prominent use of loudspeakers in mobile (fixed to a car) or fixed (in a market) form were just as significant to understanding communicative ecologies, information flows and other issues such as trusted and accessible sources (Slater, 2013; Slater and Tacchi, 2004). 
For EAR it is important to understand communicative ecologies because any 'new' connections and networks (social and technical) that are desired by an ICT4D initiative will not happen in a vacuum, and are more likely to succeed if they are interconnected with existing, locally appropriate systems and structures. Access to ICT is not enough to ensure use (Gurstein, 2003): this can only be achieved if it is appropriated and localized in meaningful ways by local communities within their local context, grounded in the realities of the everyday lives of individuals and community groups (Tacchi, forthcoming; Tacchi, Kitner and Crawford, 2012) within the social and economic conditions in which they are situated (Keeble, 2003). It is likely to be ineffective to supply new or traditional media technologies, or training in how to use them, without taking account of how they might fit into existing communicative ecologies and opportunities and barriers.

Each community setting is complex, and each media or ICT initiative, event and relationship has a possibility of changing and shifting power relations at both individual and community level. The concept of the communicative ecologies then is not fixed, but shifting, and EAR takes this into account so that an ICT initiative can adapt and respond to changing environments, changing needs and opportunities as they present themselves.

Through EAR training researchers are asked to map their own communicative ecologies and think about what factors affect the communication choices they make. Where do they get different kinds of information, how do they share information, and how is this different for other people they know? EAR researchers are asked to map their own social network by drawing a rich picture (Monk and Howard, 1998) of the people, activities, relationships and media they are linked to on a weekly basis and to indicate the different sorts of information they get from different people and places: health, education, entertainment, family, social 
events, local news and national news. They are asked to think about the different factors that place them in different social networks.

This exercise serves to demonstrate the differences amongst people who may think initially that they are similar. Gendered differences, the availability or lack of infrastructures, the differences between urban and rural settings, differing economic states, as well as the impact of differential pricing structures are among the issues that often emerge from this exercise. Through this process, and the concept of communicative ecologies EAR researchers are able to start to see the importance of generating locally meaningful categories for their research, and for the ICT initiative they work within.

\section{Core components of EAR}

Ethnographic action research combines three research approaches: participatory techniques, ethnography and action research (ear.findingavoice.org). Participatory techniques are used to help researchers and participants understand complex issues in an inclusive and participatory manner (Chambers, 2012). Ethnography has traditionally been used to understand different cultures in detail, and reject preconceived ideas and categories in favour of locally emergent ones. Action research allows us to turn what we learn from research into actions, research those actions and adjust and adapt accordingly.

The key questions that guide EAR are:

1. What are we trying to do?

What is the purpose or goal of a project? What are its objectives? Defining the purpose of a project and specific objectives is important both for planning activities and assessing whether they have been successful.

2. How are we trying to do it? 
How is a project trying to achieve its purpose and objectives in its day-to-day operations? This requires an awareness of the practices of those involved in the project, and of its internal structures and systems, including the ways in which resources are used. It emphasizes the need to not only research the communities the project is trying to serve, but the internal workings of the project itself, maintaining a high degree of reflectivity and self-awareness.

\section{How well are we doing?}

The third question demands a realistic and researched evaluation of how a project is working to achieve its purpose and objectives. EAR uncovers how well a project is doing according to local communities, local users, project staff and volunteers, donors and other external agencies.

\section{How can we do it differently/better?}

Informed by research findings, the fourth question requires a re-evaluation of purpose and objectives, a review of processes and practices, and an analysis of effectiveness, achievements and shortfalls. It requires renewed planning and actions that draw on the research, reflection and evaluation undertaken in order to improve the overall effectiveness and sustainability of the project. This whole process should allow the development and ongoing adjustment of short-, medium- and long-term plans.

EAR proposes that these four questions should be asked periodically. In answering them, projects are effectively being researched, evaluated and developed, continuously. The processes of EAR assist in asking these key questions and in defining more specific questions appropriate to each project and its purpose. Both broad and targeted research continues throughout the life of the project contributing to an ongoing research process of: 
Planning research $\rightarrow$ conducting research (collecting and documenting data) $\rightarrow$ organizing coding and analysing data $\rightarrow$ planning and action

\section{Key tools or methods used in EAR projects}

A range of key methods or 'tools' are used by EAR researchers to uncover and explore different kinds of knowledge. Multi methods are encouraged because this is likely to open up different perspectives and different data on research topics and likely to lead to more robust findings. Each research plan therefore should use at least three tools from the EAR 'toolbox' (http://ear.findingavoice.org/toolbox/index.html). The key methods include:

Participatory techniques: Techniques such as mapping, sequencing and comparing are aimed at getting EAR researchers started in collecting data and quickly gaining initial understandings of the local area, local people and local issues - including local communicative ecologies. While they are a useful way of starting EAR work, they can also be drawn on at any time to explore issues in different ways, and to test findings or ideas generated using different tools. They can also be used for consensus building.

Observation, participant observation and field notes: This is the kind of data collecting activity that EAR researchers continuously undertake, and can also be undertaken by anyone involved in an initiative simply by reflecting on what they observe and recording this in the form of field notes. This is encouraged by EAR researchers as they work towards developing a research culture. Field notes record as much as possible of what EAR researchers see and hear and also record their own reactions and ideas as they happen.

In-depth interviews: In the EAR context these are detailed conversations with a range of people, guided by an interview schedule - a list of a few major topics to be covered in each interview - while leaving sufficient room to respond to what is interesting in the conversation. 
Short questionnaire-based surveys: All of the tools above generate detailed information on a small number of participants. Short questionnaire-based surveys can allow researchers to generate less detailed information from larger numbers of people and to collect relevant quantitative data.

Diaries, feedback mechanisms and other 'self-documentation': All kinds of participants - staff, users, and community members - can express themselves on a range of social or personal issues; keep logs of their activities; or document their lives through text, audio recordings, photographs or drawings. Centres can also use feedback forms, visitors' books, log-books, suggestion boxes, and other ways to obtain feedback.

The online EAR handbook contains examples of the ways in which these methods were used in combination with the key approaches in the Finding a Voice project, described in the next section.

\section{Further applications of EAR}

It should be noted that EAR was developed to be useful and useable by local ICT4D initiatives and researchers from a range of backgrounds and varying levels of education. EAR does not assume any previous training in any of the social sciences. It is not designed for external evaluators but for local embedded researchers. In the research projects described here external (academic) researchers overseeing the training and application played an important role, but in theory at least, once adequately trained anyone can use EAR. EAR researchers trained through the ictPR project went on to train and supervise EAR researchers in the Finding a Voice project.

\section{Finding a Voice}

One of the interesting findings from the ictPR project was that the most encouraging engagement with the ICT interventions and media technologies came about when people were able to 'make' content themselves. This led to an interest in participatory content 
creation, with an emphasis on locally produced and meaningful content, and to issues of voice. Finding a Voice: Making Technological Change Socially Effective and Culturally Empowering was a three year research project supported by the Australian Research Council and UNESCO and UNDP. It presented an opportunity to expand EAR over a longer duration starting in 2006, and took place across local community media and ICT initiatives in India, Nepal, Sri Lanka and Indonesia. Finding a Voice had two main strands: to develop participatory content creation activities appropriate to each initiative, and to further develop and embed EAR.

A network of 15 ICT centres (or group of centres) was set up across the four countries. Part of the project aimed to explore how different combinations of media work together. A central research question was: 'Can old and new ICTs be used to give otherwise marginalized people a voice, and if so, who will listen?' We worked with the centres to explore and experiment with a range of participatory content creation ideas and activities that could involve a range of participants including the most marginalized (Tacchi and Grubb, 2007). The first of several content creation training workshops was on digital stories, which all of the centres were able to make using computers, photographs and storytelling. Each centre explored and adapted this format in interesting ways (Tacchi, 2009). Local EAR researchers were trained to inform and document these content creation activities. They were trained through EAR workshops and further supported online and face-to-face through field visits by an Australian team of researchers. Some of the EAR researchers who had been a part of ictPR became peer trainers and coordinators for the new researchers. An online portal allowed them to share their experiences and research findings, and allowed us to provide feedback, ask questions, and compare and contrast what was happening in each site. 
The findings from the research project are many, and documented in a range of publications (Tacchi and Kiran, 2008; Tacchi, 2012; Watkins and Tacchi, 2008), and on the Finding a Voice website (http://findingavoice.org). Of relevance here is the learning from Finding a Voice for the development of the EAR online training website, which includes examples of data and research practices from the local researchers. It also contains comments and tips from them, which demonstrate some of the ways in which the core concept of communicative ecologies and the ethnographic and action research principles are understood by them (http://ear.findingavoice.org/started/5-0.html):

To be an EAR researcher it is necessary to work as a part of the community. Try to live life like the people in your research area. (Atul)

I would encourage the selection of one of your research areas for onsite training, giving more practical experience of EAR research in the community. (Deepak)

While EAR should take note of the views of all the stakeholders in a given situation, it should give weight to those who are excluded and marginalized. An EAR researcher is one who goes to the field as a learner and listener, with a feeling of humility. The results of ethnographic action research are to be validated by the people themselves. (Jancy)

Please present yourself openly and honestly to the community people. Always give value to the community's ideas and knowledge. Be aware, if your work is not really helpful for the local community it is meaningless for them. So you have to first target to real grass root people. (Sita)

You should build up a rapport with the community and be in touch with the community so that they can be involved in the research and in your initiative's activities. Find the key informants who can help you in the field. You should also participate in their festivals and this will help you to meet many people. (Srinivas) 
Use a range of different research tools to give you more data from different angles. (Govinda)

EAR was picked up by a range of other researchers and organizations, including Equal Access, an international communication for development (C4D) NGO. They requested a series of EAR workshops for Equal Access Nepal (EAN) because they wanted to build their internal capacity to deepen their understanding of the impact of the radio content they produced and distributed across Nepal. EAR was found to be useful, but EAN found it hard to apply it consistently or systematically - they wanted to use it to improve their own practice, but also needed to prove impact to donors, and because of this we started to collaboratively developed the idea for the research project Assessing Communication for Social Change (AC4SC), a four-year project that began in 2007.

\section{Application of EAR for participatory monitoring and evaluation}

The idea was to develop EAR into a more explicit monitoring and evaluation and impact assessment methodology embedded in Nepal's EAN. Previous uses of EAR as described above relied on one or two EAR researchers working within and attempting to influence an organization (Tacchi and Kiran, 2008). Here the idea was to transform the organization by establishing evaluative systems and processes within it. Where EAR had grown in earlier research as a project development methodology, here we sought to transform and extend it into a strong and participatory evaluation methodology.

We worked with Nepalese EAN staff, following a participatory action research approach, to develop systems and processes to assess the impacts of two popular community radio programs: Saathi Sanga Manka Khura ('chatting with my best friend') and Naya Nepal ('New Nepal'). Through this collaboration EAN's monitoring and evaluation team developed research plans, built and trained a network of community researchers, collected and organized 
data from sites across Nepal, developed systems of analysis, and reported regularly to content teams (producers) and management. Overall an EAR approach was maintained, and communicative ecologies continued to be important, but here it was the monitoring and evaluation staff who oversaw and analysed data collection, much of which was gathered by a team of 'community researchers' whom they trained and managed.

Because the community researchers were part time (often students) and their role in the larger system was to collect and send data and not to be intimately involved with the analysis (although they were expected to send their thoughts and insights) we developed a monitoring and evaluation staff/community researchers' manual that included a 'lite' version of EAR. Before a community researcher was recruited the team undertook a scoping study of each location, exploring local issues, demographics, political and social structures, and including a communicative ecologies analysis. This gave EAN an initial sense of local communicative practices before starting to work on the ground. While the staff and community researchers generated data, EAN set up systems and processes for managing and analyzing it. A key challenge initially was to deal with large amounts of text based or diagram based (through participatory techniques) data, and a rigorous process for its analysis.

Towards the end of the four years, a participatory monitoring and evaluation toolkit was produced collaboratively with EAN. This will be described in the concluding section.

\section{Conclusions}

All of the research discussed above and the development of EAR itself were driven by a pragmatic need. They were funded in order to develop useful methodologies for developing effective communication and ICT4D initiatives and evaluating them in useful and useable ways. There are two key online training resources available resulting from this work. 
For EAR there is the online handbook developed in 2007 through the Finding a Voice project which can be found at ear.findingavoice.org and is useful for project development and local evaluation of media, communication and ICT for development initiatives, although it can be adapted and used for other kinds of activities and organizations. The communicative ecologies concept is at its core, because 'everyday life is made up of many different communicative resources which messily combine or conflict, that are networked with some entities but block others' (Slater, 2013). EAR is built on the idea that the success of media, communication and ICT for development initiatives depends on understanding this messiness and looking for communicative opportunities.

For the adaptation of EAR into a monitoring and evaluation methodology in the project the Equal Access Participatory Monitoring and Evaluation Toolkit can be found on the BetterEvaluation website, at betterevaluation.org/toolkits/equal_access_participatory_monitoring. Here EAR is transformed and expanded into a methodology designed to help C4D organizations demonstrate impacts and outcomes of their initiatives, to listen to their listeners, continuously learn, and feed this learning back into the organization and its practices. It retains the essence and principles of EAR, but adapts it into a monitoring and evaluation approach. The toolkit was developed with EAN and is modular. It includes modules on effective communication, feedback and reporting systems in a PM\&E process; setting objectives and indicators; research and Participatory Monitoring and Evaluation methods; critical Listening and feedback sessions; doing qualitative data analysis; and, getting started and planning for Participatory Monitoring and Evaluation and impact assessment. It also includes the CR (community researchers') Manual (incorporating a 'lite' version of the EAR handbook). 
Both of these resources emerge through practice and in response to the expressed needs of the organizations involved. They are also designed to be adapted for use in other situations. The research projects described above, and the learnings from them, have contributed most recently to the development of a framework for evaluating communication for development (Lennie and Tacchi, 2013; Tacchi and Lennie, 2014). There are seven key components underpinning this framework: participatory, holistic, critical, realistic, learning-based, emergent and complex (see Figure 22.1).

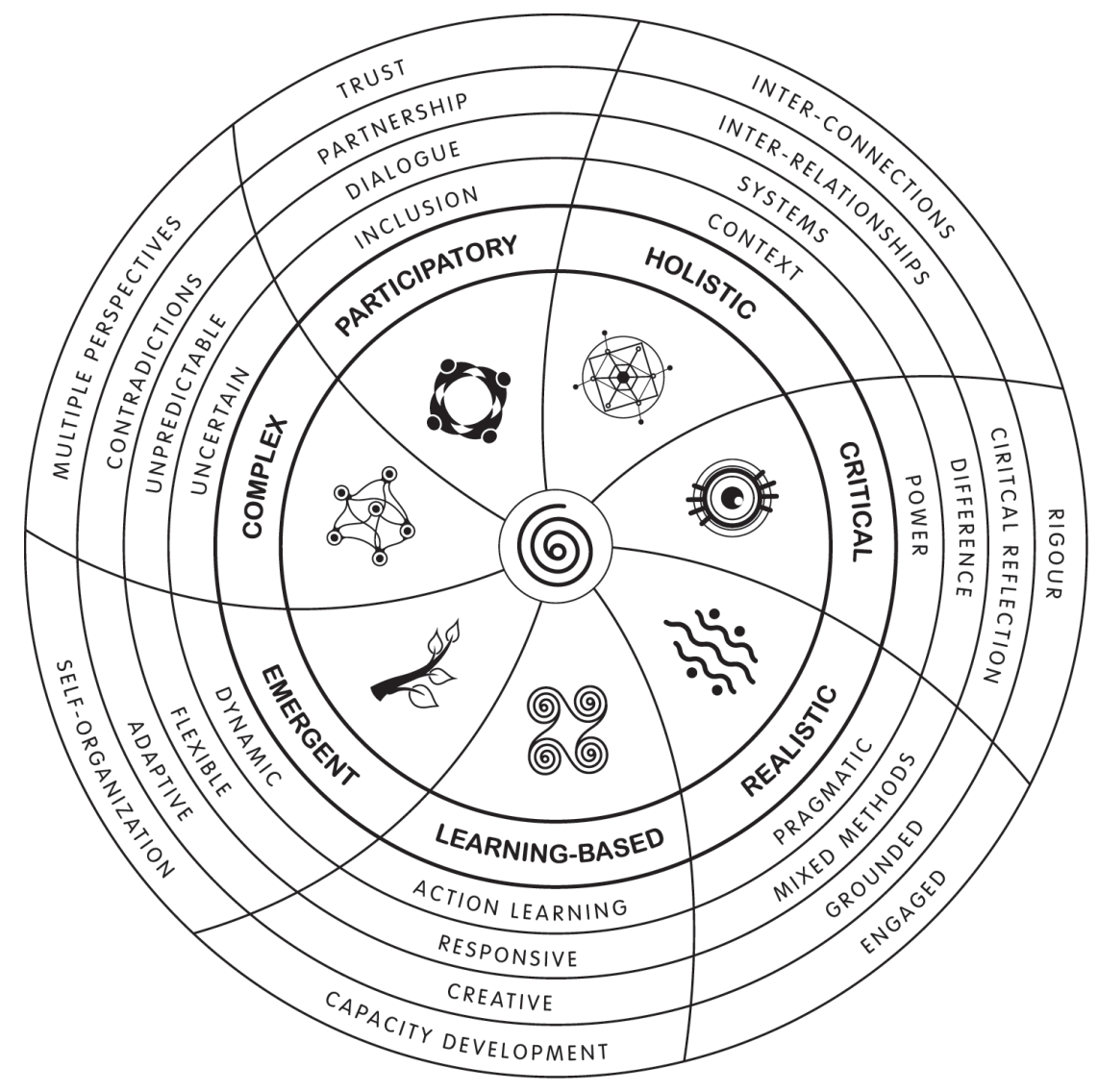

Figure 22.1 Key components in the framework for evaluating C4D from Lennie and Tacchi (2013)

These components, and the framework, effectively captures, and was indeed partly informed by the experience of developing ethnographic action research, and serves as a neat way of summing up the main principles of the EAR approach. 


\section{Acknowledgements}

Numerous people have been involved in the development of EAR. They are too numerous to list here, but acknowledgements can be found on the EAR training website: http://ear.findingavoice.org/info/credits.html. I do however single out and acknowledge the original and foundational contributions of Don Slater and recommend his recent book, New Media, Development and Globalization (2013) for a detailed explanation of the communicative ecology concept and the KCRIP study and ictPR project.

The British Government's Department for International Development (DFID) funded the research project in Sri Lanka which was led by Don Slater with Jo Tacchi and Peter Lewis, and local research assistants Lasanthi Daskon and Tanya Notley.

The study 'Assessing Communication for Social Change: A New Agenda in Impact Assessment for Communication for Development Initiatives' was funded by the Australian Research Council and Equal Access. The research team comprised Jo Tacchi, June Lennie, Andrew Skuse and Michael Wilmore.

\section{References}

Chambers, R. (2012) Provocations for Development. Rugby: Practical Action Publishing.

Gurstein, M. (2003) 'Effective use: A community informatics strategy beyond the Digital

Divide' First Monday, Volume 8, Number 12. Available at:

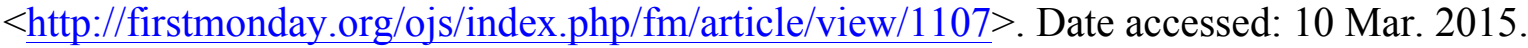

Keeble, L. (2003). Why Create? A Critical Review of a Community Informatics

Project. Journal of Computer-Mediated Communication, 8 (3) Available at http://onlinelibrary.wiley.com/doi/10.1111/j.1083-6101.2003.tb00213.x/full. Date accessed: 10 Mar. 2015. 
Lennie, J. and Tacchi, J. (2013). Evaluating Communication for Development: A Framework for Social Change. Abingdon: Routledge.

Monk, A., \& Howard, S. (1998). The Rich Picture: A Tool for Reasoning about Work Context. ACM SIGCHI interactions, 5 (2), 21-30.

Patton, M.Q. (2011). Developmental Evaluation: Applying Complexity Concepts to Enhance Innovation and Use. New York: Guilford Press.

Slater, D. (2013). New Media, Development and Globalization: Making Connections in the Global South. Cambridge: Polity Press.

Slater, D., Tacchi, J. and Lewis, P. (2002). Ethnographic Monitoring and Evaluation of Community Multimedia Centres: A Study of Kothmale Community Radio Internet Project, Sri Lanka. London: DFID.

Slater, D. and Tacchi, J. (2004). Research: ICT Innovations for Poverty Reduction. New Delhi: UNESCO.

Tacchi, J. (2014). 'Being meaningfully mobile: Mobile phones and development'. In Servaes, J. (ed.), Technological Determinism and Communication for Sustainable Social Change. Lanham, MD: Lexington Books, pp. 105-124.

Tacchi, J. (2012) 'Open content creation: the issues of voice and the challenges of listening' New Media and Society 14(4) 652 - 668.

Tacchi, J. (2009). 'Finding a Voice: Digital Storytelling in Southeast Asia.' In Hartley, J. \& McWilliam, K. (Eds.) Story Circle: Digital Storytelling Around the World. Oxford: WileyBlackwell, pp. 167-175. 
Tacchi, J. and Grubb, B. (2007). 'The Case of the e-tuktuk'. Media International Australia incorporating Culture and Policy 125. Pp. 71-82.

Tacchi, J. and MS Kiran. (Eds.) (2008) Finding a Voice: Themes and Discussions. New Delhi. UNESCO.

Tacchi, J., Kitner, K. and Crawford, K. (2012). 'Meaningful mobility: Gender, development and mobile phones', Feminist Media Studies, 12(4), 528-537.

Tacchi J and Lennie J (2014). 'A participatory framework for researching and evaluating communication for development and social change'. In Wilkins, K.G., Tufte, T. and Obregon, R. (eds), The Handbook on Development Communication and Social Change. Oxford: Wiley Blackwell, pp. 298-320.

Tacchi, J., Slater, D. and Hearn, G. (2003). Ethnographic Action Research Handbook. New Delhi: UNESCO.

Watkins, J. and Tacchi, J. (Eds.) (2008) Participatory Content Creation for Development: Principles and Practices. New Delhi. UNESCO. 